\title{
Frustration tuning and perfect phase synchronisation in the Kuramoto-Sakaguchi model
}

\author{
Markus Brede \\ Complexity, Agents and Interactions, \\ Department of Electronics and Computer Science, \\ University of Southampton, United Kingdom \\ Alexander C. Kalloniatis \\ Defence Science and Technology Group, \\ Canberra, Australia 2600.
}

(Dated: June 1, 2016)

\begin{abstract}
We present an analysis of conditions under which the dynamics of a frustrated Kuramoto - or Kuramoto-Sakaguchi - model on sparse networks can be tuned to enhance synchronisation. Using numerical optimisation techniques, linear stability and dimensional reduction analysis, a simple tuning scheme for setting node specific frustration parameters as functions of native frequencies and degrees is developed. Finite size scaling analysis reveals that even partial application of the tuning rule can significantly reduce the critical coupling for the onset of synchronisation. In the second part of the paper, a co-dynamics is proposed which allows a dynamic tuning of frustration parameters simultaneously with the ordinary Kuramoto dynamics. We find that such co-dynamics enhance synchronisation when operating on slow time scales, and impede synchronisation when operating on fast time scales relative to the Kuramoto dynamics.

PACS numbers: 05.45.Xt, 05.70.Ln, 89.75.Fb, 89.75.Hc
\end{abstract}

\section{INTRODUCTION}

The Kuramoto model of coupled oscillators [1] has proven an enduring basic model of complex systems, particularly in its extension to general networks, see [2-5] for recent reviews. Application of the model are found in a variety of contexts, ranging from arrays of Josephson junctions [6] to the power grid [7] or biological oscillations found in populations of yeasts [8] and various other types of biological rhythms, cf., e.g., [9, 10]. This model consists of oscillators $\theta_{i}$ at nodes $i$ of an undirected network of size $N$ given by an adjacency matrix $A_{i j}$, assigned native frequencies $\omega_{i}$ that are typically drawn from some distribution such as uniform, Gaussian, Lorentzian or generally unimodal symmetric. Through the dynamics in the system of equations

$$
\dot{\theta}_{i}=\omega_{i}+\sigma \sum_{j} A_{i j} \sin \left(\theta_{j}-\theta_{i}\right),
$$

after some critical coupling $\sigma_{c}$ a macroscopic fraction of the oscillators spontaneously phase synchronise $\theta_{i} \approx \theta_{j}$ to a collective frequency given by the average $\bar{\omega}$ over the frequency ensemble. A variation of this model is the Kuramoto-Sakaguchi model [11] where a global shift $\lambda$ - or frustration - is introduced in the sine function. In this paper we show that allowing localised frustrations $\lambda_{i}$, namely

$$
\dot{\theta}_{i}=\omega_{i}+\sigma \sum_{j} A_{i j} \sin \left(\theta_{j}-\theta_{i}+\lambda_{i}\right),
$$

and tuning the $\lambda_{i}$ in relation to the frequencies $\omega_{i}$, or alternately providing a codynamics for them, allows im- proved synchronisation to a different collective frequency than $\bar{\omega}$ to be achieved, and at lower coupling.

In the limit of all-to-all coupling $A_{i j}=1$ for all $i, j$ and $N \rightarrow \infty$, the model Eq. (1) allows for an analytical solution [1]. Inspired by Watts and Strogatz' introduction of small world networks [12], the literature has strongly focussed on analysing the model on sparse complex networks $[4,5]$. A particular interest in this line of thinking has been to understand conditions under which synchronisation can be improved, e.g., determining the types of correlations of native frequencies along the links of a network that can strongly influence overall synchronisation behaviour [13-18].

The global Kuramoto-Sakaguchi model with large $N$ and all-to-all coupling is similarly soluble for the critical coupling [11]. Some variations of the model consider random pinning fields [19] while in others the frustrations are either global or random for every node [20, 22], or allocated according to a multi-network structure for interacting populations $[23,24]$. While such additions introduce additional degrees of disorder to the traditional Kuramoto dynamics, we are interested in whether frustrations may be tuned to enhance synchronisation. We indeed find this to be the case, to a degree even more than an equilibrium analysis reveals, where enhancement occurs in two ways: macroscopic synchronisation is found at lower values of critical coupling than for the normal Kuramoto system of Eq. (1); and this synchronisation occurs at collective frequencies $\Omega$ which may be different from (both less and greater than) $\bar{\omega}$, something already observed for the global Kuramoto-Sakaguchi model $[17,20]$. Synchronisation in this setting requires specifically tuned frustration parameters and one might wonder 
how appropriate configurations of such parameters may be reached in real world systems. By introducing a codynamics in which frustration parameters evolve simultaneously with the normal Kuramoto dynamics, we illustrate a simple dynamical scheme via which tuned configurations may be arrived at. The suggested tuning scheme follows a similar approach to earlier work on adaptive coupling schemes between coupled oscillators [21]. We propose that, in adaptations of the Kuramoto model to social/technological systems with multiple heterogeneous agents, for example [25, 26] (see [5] for an expanded list of such applications) these mechanisms of tuned frustrations and codynamics may offer new approaches to enhanced or more efficient cooperative behaviour.

The paper is organised in two main parts. Firstly, we examine the case of static frustration parameters in which we study the steady state solutions and then examine the system numerically, particularly using an optimisation scheme for tuning the parameters. We see there that enhancement of synchronisation consistent with the steady state analysis is possible. We supplement insights from this analysis by calculating a critical condition for transitions using a dimensional reduction approach. Subsequently, we undertake finite size scaling analysis which demonstrates that tuning allows for an onset of synchronisation for lower coupling than for the ordinary $\mathrm{Ku}-$ ramoto model and that partial application of the rule to a fraction of oscillators gradually improves synchronisation. In the second part we introduce a co-dynamics that allows co-evolution of the frustration parameters with the Kuramoto dynamics. We then demonstrate that such a simple evolution scheme can tune the frustration parameters to enhance synchronisation and we analyse under what conditions this is the case. The paper concludes with a summary and discussion of results.

\section{STATIC KURAMOTO-SAKAGUCHI SYSTEM}

\section{A. Steady state analysis}

To understand the basic properties of the local Kuramoto-Sakaguchi model we repeat the steady state solution considered for the global case, such as in [17]. We consider solutions of the form

$$
\theta_{i}(t)=\Omega t+\phi_{i}(t)
$$

where $\phi_{i}$ is regarded as a small fluctuation allowing for expansion up to linear terms, namely $\left(\phi_{i}\right)^{2} \approx 0$. Inserting this ansatz into Eq. (2) we extract the equation with terms $O\left(\left(\phi_{i}\right)^{0}\right)$ :

$$
\Omega=\omega_{i}-\sigma k_{i} \sin \lambda_{i}
$$

from the $\theta_{i}$ dynamics, where $k_{i}=\sum_{j} A_{i j}$ denotes the (in-)degree of node $i$. The corresponding equation linear in the small fluctuations $\phi_{i}$ is

$$
\dot{\phi}_{i}=-\sigma \sum_{j} \cos \lambda_{i} L_{i j} \phi_{j},
$$

where $L_{i j}=\delta_{i j} k_{i}-A_{i j}$ is the combinatorial Laplacian whose presence in such fluctuation problems is wellknown [17]. Multiplying by the $\cos \lambda_{i}$ gives a weighted version of the Laplacian. The spectral property of such a Laplacian - that it is positive semi-definite with a degeneracy of the zero eigenvalue corresponding to the number of components of the graph [27] - means here that the fluctuations are stable, and will decay to exactly zero, if (but not only if) all the $\cos \lambda_{i} \geq 0$. We will focus on the fluctuations in more detail later in the presence of co-dynamics. Noting that $\lambda= \pm \frac{\pi}{2}$ represent marginal stability - thus we do not allow for 'repulsive interactions' in the sense of [31] - for now we use the resulting constraint on the $-\frac{\pi}{2}<\lambda_{i}<\frac{\pi}{2}$, and focus on the equilibrium configurations.

Summing Eq. (4) over $i$ and dividing by $N$ gives

$$
\Omega=\bar{\omega}-\frac{\sigma}{N} \sum_{i} k_{i} \sin \lambda_{i}
$$

and this shows that without specific tuning of $\sum_{i} k_{i} \sin \lambda_{i}$ to equal zero then one generally has $\Omega \neq \bar{\omega}$. Thus collective frequencies other than the mean of the native frequencies can be achieved in the presence of the frustration. This is related to the property that Eq. (2) enjoys the same translation symmetry as the ordinary Kuramoto model $\theta_{i} \rightarrow \theta_{i}+\Psi t, \omega_{i} \rightarrow \omega_{i}+\Psi$ for any real $\Psi$ giving the freedom to fix one integration constant. The contrast here is that the conserved charge no longer follows from $\sum_{i} \dot{\theta}_{i}=0$ that is satisfied by Eq. (1); rather, the charge for the Kuramoto-Sakaguchi case is not a closed form functional of $\theta$.

Choosing some $\Omega$ now, the $N$ equilibrium conditions of Eq. (4) can be rewritten

$$
\sin \lambda_{i}=\frac{\Omega-\omega_{i}}{\sigma k_{i}},
$$

which represents a means of tuning the frustrations to achieve a collective frequency $\Omega$. Note that for $\sigma$ increasingly large, the frustrations $\lambda_{i}$ become small so that perfect synchronisation requires fine-tuning to vanishing values. If there is any noise in practice, then tuning becomes difficult and perfect synchronisation becomes impossible leading to "erosion" effects [28, 29].

Observe now that because of the boundedness of the sine in Eq. (7), solutions will exist only for

$$
\frac{\left|\Omega-\omega_{i}\right|}{\sigma k_{i}} \leq 1
$$

We therefore refer to Eq. (8) as the recruitment condition. We may also use Eq. (8) to obtain a threshold coupling condition such that all oscillators across the network may be recruited into a cluster oscillating at the 
chosen collective frequency $\Omega$ :

$$
\sigma_{r}=\max _{i} \frac{\left|\Omega-\omega_{i}\right|}{k_{i}} .
$$

We note that no analogue to the threshold coupling, as emerges from Eq. (8), exists as a sharp condition in the ordinary Kuramoto model: the best one can do is demand some consistency for the linearisation approximation to hold $[33,34]$. In this regime, the order parameter $r$ defined via

$$
r(t) \exp (-i \psi(t)) \equiv \frac{1}{N} \sum_{j} \exp \left(-i \theta_{j}(t)\right)
$$

may be expanded in fluctuations $\phi_{i}$, as

$$
\begin{aligned}
r^{2} & =\frac{1}{N^{2}} \sum_{i, j} \cos \left(\phi_{i}-\phi_{j}\right) \\
& \rightarrow 1
\end{aligned}
$$

as $t \rightarrow \infty$ if $\cos \lambda_{i} \geq 0$ since, then, $\phi_{i} \rightarrow 0$ from Eq. (5). This means that in this regime, the order parameter shows exact phase synchronisation as a consequence of the freedom in the $\lambda_{i}$ to tune them (or, alternately phrased, there is zero "erosion" of synchronisation in the sense of $[28,29]$ because of the freedom to tune the $\lambda_{i}$ such that the "synchrony alignment function" - see also section 7 of [30] - exactly vanishes). The threshold coupling $\sigma_{r}$ for all oscillators to be recruited in this way must be distinguished from the critical coupling $\sigma_{c}$ usually defined as value where the ensemble or time average of the order parameter $r$, deviates from zero. This is typically far from the regime where linearisation will hold. We must therefore resort to numerical simulation to examine the critical coupling $\sigma_{c}$. In particular, we explore what choices of the frustration parameters enhance synchronisation for $\sigma<\sigma_{c}$, and demonstrate that even partial tuning of frustration parameters in ensembles of coupled oscillators can significantly enhance synchronisation.

\section{B. Optimisation Experiments}

We now examine numerically whether the system of Eq. (2) allows for enhanced synchronisation when the frustration parameters are selected as above, and what occurs when only a subset of oscillators satisfy the recruitment condition. To this end we consider a numerical optimisation scheme for a given network with adjacency matrix $A_{i j}$ and given configuration of native frequencies $\omega_{i}$ drawn uniformly at random from $[-1,1]$. We seek settings of the static frustration parameters $\lambda_{i}$ that optimise synchronisation. Throughout the paper we solve the dynamics for quite a variety of networks (random, random regular, scale free and small world), and network sizes $(N=50,100,1000,10000)$. Each choice tests certain aspects of the frustrated and codynamics models in a targeted way, while demonstrating the ubiquity of

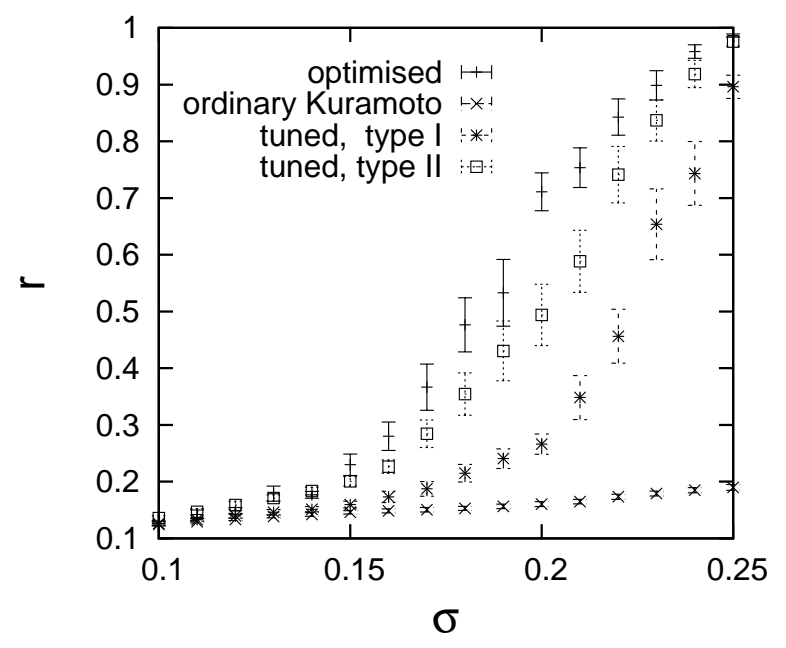

FIG. 1. Results from numerical simulations of the average order parameter $r$ at fixed values of coupling $\sigma$ for variations of the frustrated Kuramoto model on regular random graphs with $k_{i}=4$ for each node and $N=100$. Each data point represents an average over 10 optimised configurations. Starting from the lowest curve, we compare: (a) the ordinary Kuramoto dynamics with $\lambda_{i}=0$, namely Eq. (1) (b) type I, the frustrated Kuramoto(-Sakaguchi) dynamics, Eq. (2), with frustration parameters set to $\sin \lambda_{i}=-\omega_{i} /\left(k_{i} \sigma\right)$ if $\omega_{i}<k_{i} \sigma$ and $\lambda_{i}=0$ otherwise, (c) type II, frustration dynamics with frustration parameters set to $\sin \lambda_{i}=-\omega_{i} /\left(k_{i} \sigma\right)$ if $\omega_{i}<k_{i} \sigma$ and $\sin \lambda_{i}= \pm 1$ otherwise. and (d) frustrated dynamics with frustration parameters determined by the optimisation procedure.

the behaviours across network types and sizes. At this stage, we consider a regular random graph where each node has degree $k_{i}=4$ and $N=100$. The degree of synchronisation is measured by the order parameter $r$ which we time-average after discarding a transient.

Similar to $[13,14]$ we use the following optimisation scheme: (i) start with some random initial condition, (ii) pick a node $i$ at random and add a phase shift randomly selected from $[-\pi, \pi]$ to its frustration parameter to obtain a modified configuration, (iii) calculate the average order parameter for the modified configuration (for initial conditions set to zero), and (vi) accept the new configuration if it gives a larger average order parameter than the previous one and reject otherwise. If a modified configuration is rejected, we revert to the last previously accepted configuration and proceed with step (ii). Experiments are run until no configuration has been accepted for $L^{2}$ trials, with $L$ the number of links of the network. Results in the optimisation are obtained for initial conditions $\theta_{i}=0$, but as observed in $[13,14]$ we find that the optimised configurations are independent of this choice.

Examining the dependence of the average order parameter on coupling in Fig. 1 gives the key result of the optimisation experiments. For each of the 10 network and native frequency configurations (sampled at random 

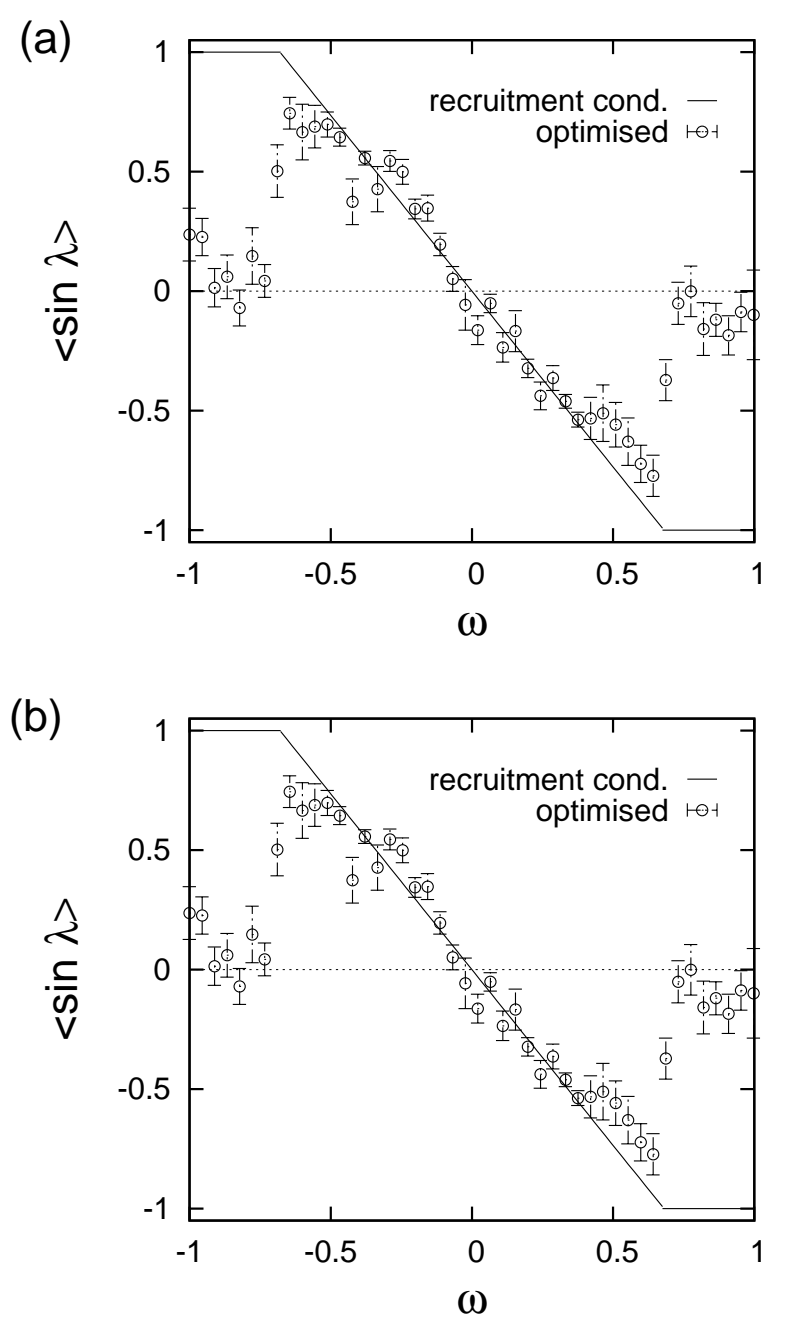

FIG. 2. Plots of the average value of $\sin \lambda_{i}$ vs native frequencies $\omega_{i}$ to check fulfilment of the recruitment condition Eq. (7), given by solid lines, for configurations evolved for $\sigma=0.17$ (a), and configurations evolved for $\sigma=0.24$ (b). The data represent averages over 10 configurations of optimized frustration parameters on regular random graphs (setting of graphs etc. are as in Fig. 1).

at the start of the optimisation procedure) we calculate the dependence of the order parameter on the coupling strength and average over different initial conditions, and then present averages over the 10 configurations. In Fig. 1 the results for the optimisation are compared to different scenarios for tuning the frustration parameters for the same network and native frequency configurations, in particular for nodes where recruitment of an oscillator, Eq. (8), cannot be implemented and where Eq. (7) does not prescribe frustration parameters for oscillators with $|\omega|>\sigma k$. For these scenarios we set $\Omega=0$ in imposing Eq. (7) and choose for the nodes that cannot satisfy this condition either $\lambda_{i}=0$, labelled 'type I', or $\sin \lambda_{i}=-\operatorname{sgn}\left(\omega_{\mathrm{i}}\right)$, denoted 'type II'. Note that this second strategy means setting non-recruited frustrations to the value giving marginal stability, as mentioned earlier, and might be regarded as a natural continuation of Eq. (7). Both extremes are included in Fig. 1; the closeness of the latter to the optimised case strongly suggests that the second choice, namely that for marginal stability in the non-recruited oscillators, is superior to achieve enhanced synchronisation. We have also explored a range of choices in-between, which smoothly interpolate between the results shown in the figure for $\sin \lambda=0$ and $\sin \lambda=-\operatorname{sgn}(\omega)$.

The first comparison in Fig. 1 is between any of the tuning scenarios (asterisks and squares) to the usual nonfrustrated Kuramoto dynamics (crosses): it is very obvious that targeted tuning of the frustration parameters significantly improves synchronisation over that for the ordinary Kuramoto model. However, comparing the optimisation results to the two tuning scenarios we see that further improvements in synchronisation are possible over and above the pure recruitment condition Eq. (7).

We explore this further in the panels of Fig. 2 in which we investigate the pattern of frustration parameters in optimized configurations. We do this by plotting the dependence of the averaged sines of the frustrations on native frequencies for two situations. The panels of Fig. 2 show solid lines that illustrate the recruitment condition of Eq. (8), which can be approximated by $\sin \lambda_{i}=-\omega_{i} /\left(\sigma k_{i}\right)$ for the case of a symmetric initial distribution of native frequencies (for which $\bar{\omega} \approx 0$ ) and $\Omega=0$. First, with coupling $\sigma=0.17$ (top panel) we have a case where a significant fraction of nodes is not synchronised to the main collective frequency. This is based on the observation that in Fig. 1 the time-averaged order parameter is well below 0.5 at this coupling. Secondly, with $\sigma=0.24$ (bottom panel) we show a case where almost all nodes can be recruited to a synchronised cluster, as seen in $r$ being close to one in Fig. 1 for this value of $\sigma$.

In panel (a) of Fig. 2 we see that, for coupling insufficient to recruit oscillators with too large or too small native frequencies, those oscillators with small native frequency on average follow a linear relationship, while the average sines of evolved frustration parameters cluster close to $\langle\sin \lambda\rangle=0$. Since oscillators with large native frequencies cannot be recruited to the synchronised cluster, their respective frustration parameters appear to drift randomly during the optimisation, resulting in an average of zero with large fluctuations. Note that for this reason the numerical optimisation experiments do not replicate the type II condition for non-recruited oscillator phases. In contrast, in panel (b) we see the appearance of an almost perfect linear relationship for evolved frustration parameters when the coupling is high. 


\section{Finite size scaling analysis and determination of the critical coupling}

Optimisation experiments in small systems backed up by equilibrium analysis presented in the previous subsections suggested that tuning the frustration parameters might allow synchronisation at lower coupling than for the ordinary Kuramoto model. In this section we carry out a finite size scaling analysis that aims to establish (i) whether this earlier finding persists in the thermodynamic limit or is an artifact of small system size, and (ii) what influence partial fulfillment of the recruitment condition has on the onset of synchronisation.

For this purpose, the frustration parameters $\lambda_{i}$ of the static model are set as follows. For each $i=1, \ldots, N$ with probability $p$ we set

$$
\sin \lambda_{i}= \begin{cases}-\omega_{i} /\left(k_{i} \sigma\right) & \text { if } \omega_{i}<k_{i} \sigma \\ -\operatorname{sgn}(\omega) & \text { if } \omega_{i} \geq k_{i} \sigma\end{cases}
$$

and $\lambda_{i}=0$ with probability $1-p$. This setting allows us to smoothly explore the effect of tuned phase shifts $\lambda_{i}$. Specifically, if $p=0$ our framework reproduces the conventional unfrustrated Kuramoto model on the network under consideration, and if $p=1$ we are in the regime in which all oscillators are tuned.

We again explore the model via numerical simulations, first by evaluating the dependence of the average order parameter on the coupling strength for three types of network and native frequencies drawn from a uniform distribution between $[-1,1]$. In Fig. 3 we give results for Erdös-Rényi (ER) random graphs (panel (a)), the Watts-Strogatz small worlds (panel (b)), and BarabasiAlbert scale free networks (panel (c)) of $N=1000$ nodes. The data clearly indicate improved synchronisation behaviour as the fraction of tuned oscillators is increased, but also highlight that a boost for synchronisation can be obtained even by tuning only a small fraction of oscillators. In comparing the enhancement of synchronisation between the three types of networks we also see that the most significant change in dependence of $r$ on $\sigma$ occurs for the small world case, where a gradual increase of $r$ becomes almost a sharp transition as $p$ increases.

Next, for a more systematic exploration of critical thresholds, we analyse the scaling of the order parameter $r(\sigma, N)$ with the system size $N$. Since the focus of the analysis is determining the critical point rather than critical exponents we follow the procedure outlined in [35] rather than more elaborate finite size scaling techniques $[36,37]$. The procedure is based on the following observations. Below the critical point oscillators rotate essentially independently and thus one expects a scaling of the order parameter $r(\sigma, N) \propto N^{-1 / 2}$ for $\sigma<\sigma_{c}$. On the other hand, for $\sigma>\sigma_{c}$ the order parameter is expected to approach a non-zero value in the limit of $N \rightarrow \infty$. In this way plots of $r(\sigma, N)$ vs $N$ for varying coupling $\sigma$ allow us to extract the critical point. This is illustrated in the panels of Fig. 4 from which we extact the critical coupling as the smallest value of $\sigma$ for which $r(N)$ does (a)
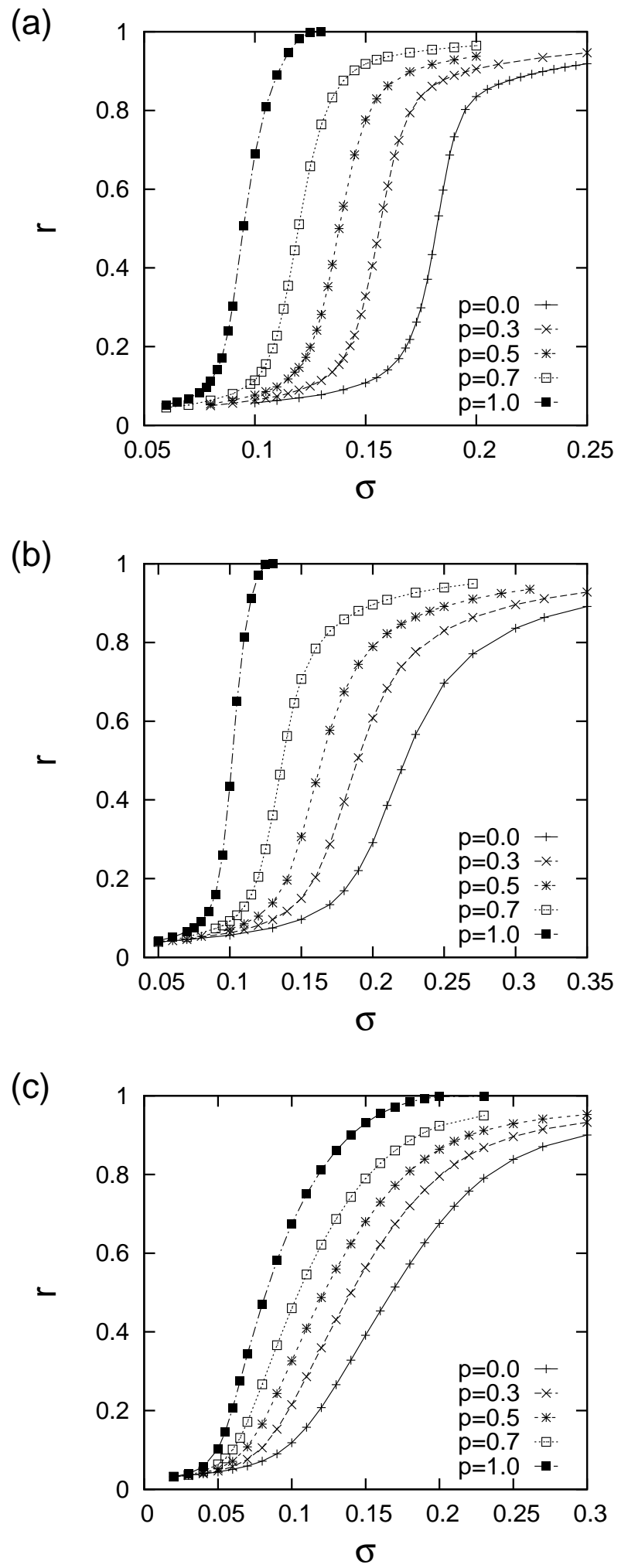

FIG. 3. Dependence of the order parameter $r$ on coupling strength for networks (with $\langle k\rangle=8$ ) of $N=1000$ oscillators with various settings of the fraction of tuned oscillators $p$. From top to bottom, data for a regular ER random graph (a), a regular Watts-Strogatz small world (with $10 \%$ shortcut density) (b), and Barabasi-Alberts scale free networks (c) are shown. Data points correspond to averages over at least 1000 random native frequency and network arrangements. 

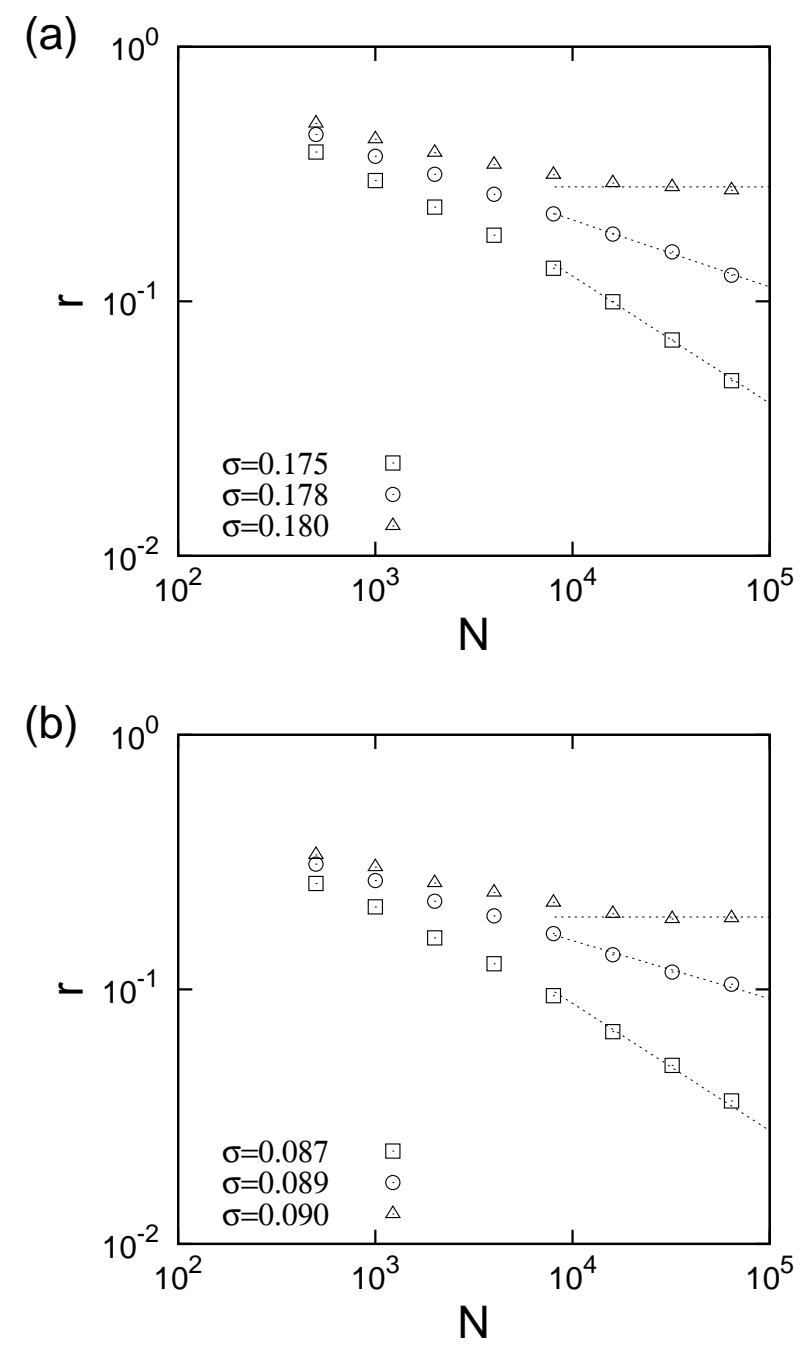

FIG. 4. Dependence of the order parameter $r$ on the system size $N$ for ER random graphs with various coupling and no tuned oscillators $(p=0$, panel (a)) and all tuned oscillators $(p=1$, panel (b)). The dashed lines indicate power laws with exponents $-1 / 2,-1 / 4$ and 0 (constant).

not converge towards a constant non-zero value as the system size is increased. For this example of an ER random graph we find $\sigma_{c}=0.178(3)$ without tuning (panel (a) of Fig. 4), and $\sigma_{c}=0.089(2)$ when all frustration parameters are tuned (panel (b) of Fig. 4). Analogous experiments for regular small worlds (with rewiring probability $p_{\text {rew }}=0.1$ ) are given in Fig. 5, showing a similar dependence of the critical coupling on the fraction of tuned oscillators. Finally, for this section, a systematic exploration of critical couplings with $p$ is shown in Fig. 6 . We observe here clearly that increasing the proportions of tuned oscillators via $p$ leads to a monotonic decrease in the critical coupling.

From these results we learn that significant enhancement of synchronisation is possible even for couplings for which, by far, not all oscillators can fulfill the recruit-
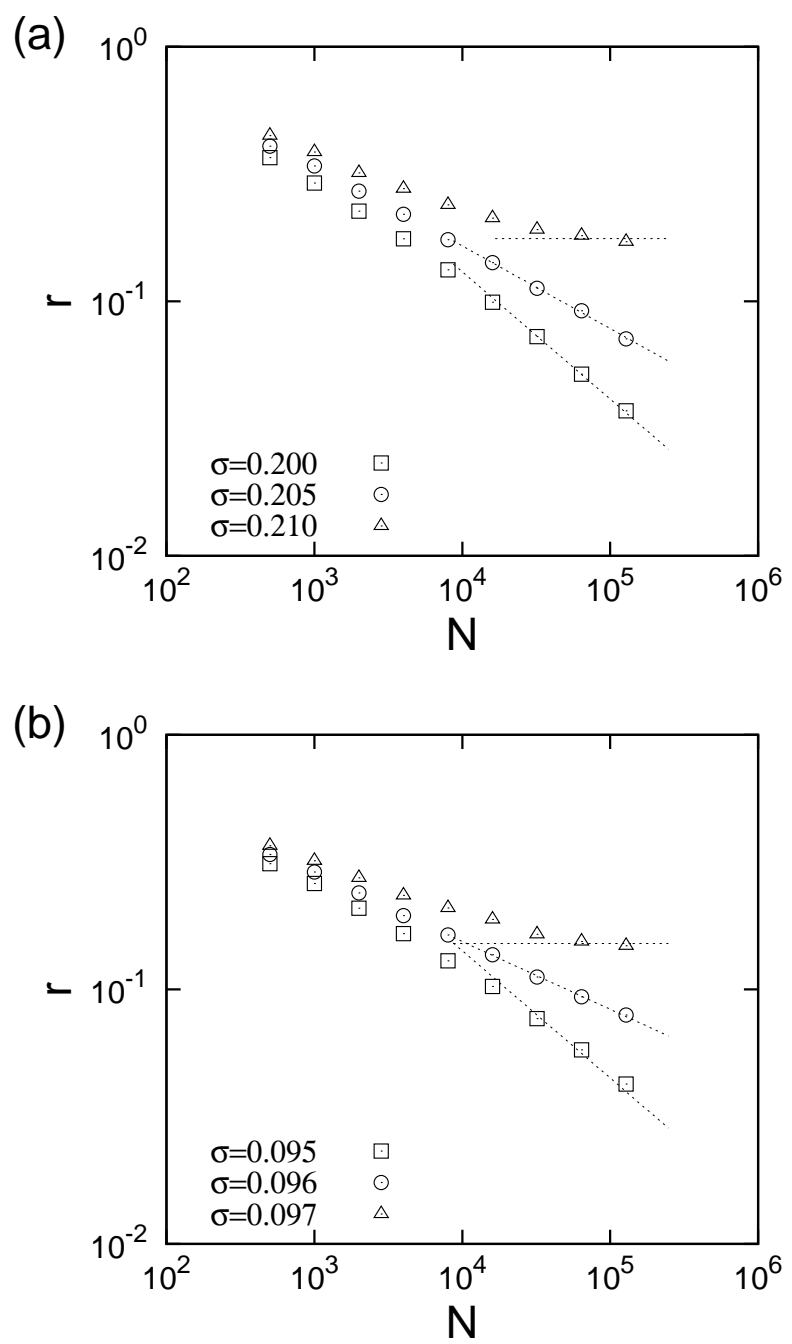

FIG. 5. Dependence of the order parameter $r$ on the system size $N$ for regular small worlds with $p_{\text {rew }}=0.1$ with various coupling and no tuned oscillators $(p=0$, panel (a)) and all tuned oscillators $(p=1$, panel (b)). The dashed lines indicate power laws with exponents $-1 / 2,-1 / 4$ and 0 (constant).

ment condition $\sin \lambda_{i}=-\omega_{i} /\left(k_{i} \sigma\right)$. This even results in an onset of synchronisation for lower coupling strengths. Moreover, when the coupling is sufficient that the recruitment condition can be fulfilled, even tuning only a fraction of the oscillators with the recruitment condition will result in enhanced synchronisation. For this reason then we see that the threshold coupling for recruitment, $\sigma_{r}$, cannot be equated with, or approximate, the critical coupling.

\section{A dimension reduction approach}

Following the idea set out in [38] we also study the Kuramoto-Sakaguchi model via a dimension reduction (or collective coordinate) approach. For this purpose, 


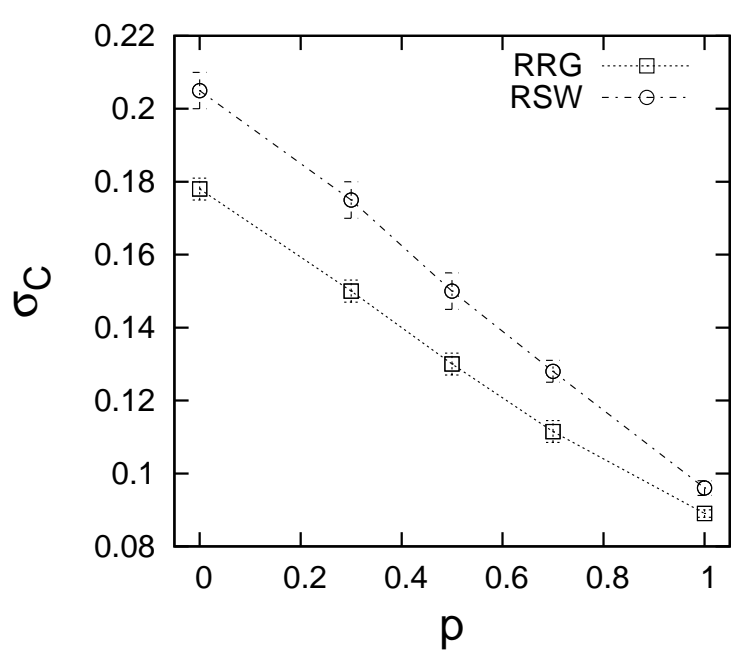

FIG. 6. Dependence of the critical coupling on $p$ for regular random graphs and regular small worlds with rewiring probability $p_{\text {rew }}=0.1$.

we apply the ansatz

$$
\theta_{i}(t)=\alpha(t) \omega_{i}
$$

which entails an error $e_{i}$ from the Kuramoto-Sakaguchi dynamics of Eq. (2), given by

$$
e_{i}=\dot{\alpha} \omega_{i}-\omega_{i}-\sigma \sum_{j} A_{i j} \sin \left(\alpha\left(\omega_{j}-\omega_{i}\right)+\lambda_{i}\right) .
$$

An equation for the evolution of $\alpha(t)$ can be found by minimising the error of Eq. (14), which is usually done by requiring that the error is orthogonal to the subspace defined by Eq. (13) which is spanned by the $\partial \theta_{i} / \partial \alpha=\omega_{i}$ and we obtain

$$
\dot{\alpha}=1+f(\alpha)
$$

with

$$
f(\alpha)=\frac{\sigma}{\sigma_{\omega}^{2}} \sum_{i} \omega_{i} \sum_{j} A_{i j} \sin \left(\alpha\left(\omega_{j}-\omega_{i}\right)+\lambda_{i}\right)
$$

and $\sigma_{\omega}^{2}=\sum_{i} \omega_{i}^{2}$

Gottwald [38] has shown that the onset of synchronisation in the unfrustrated Kuramoto model can be understood from the point at which stationary solutions $\alpha^{*}$ of Eq. (15) appear when the coupling is changed. For the unfrustrated model, [18] noticed that information about the position of $\alpha^{*}$ may be inferred by studying $1+f(\alpha)$ at $\alpha=0$. Specifically, $f(0)=0$ is independent of the system configuration (frequencies) and hence [18] (who define $f$ slightly differently from Eq. (16)) constructed an argument that, independent of coupling strength, optimal synchronisation of the system should be obtained when $f^{\prime}(0)$ is minimized - in other words, the slope of $f$ at $\alpha=0$ should be negative and as steep as possible to allow for an $\alpha^{*}$ as close as possible to zero.
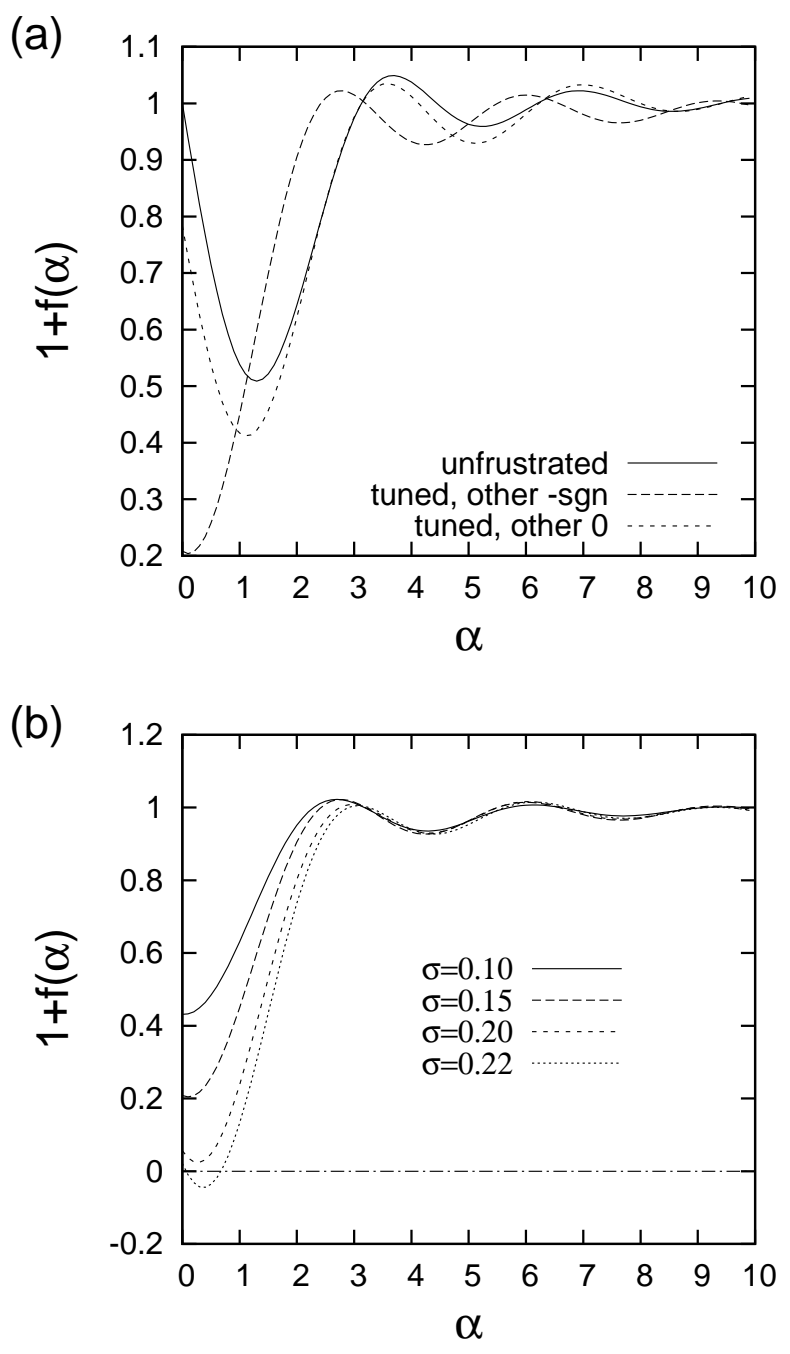

FIG. 7. (a) Comparison of the function $1+f(\alpha)$ for various tuning schemes at $\sigma=0.15$ for one realisation of a configuration of $N=10000$ oscillators on a regular small world with $k=4$, unfrustrated $\left(\lambda_{i}=0\right)$, frustrated type I (tuning recruitable oscillators according to Eq. (7) and setting $\lambda_{i}=0$ for all other oscillators), and frustrated type II (tuning recruitable oscillators according to Eq. (7) and setting $\sin \lambda_{i}=-1$ for all others). (b) All oscillators are tuned according to the third scheme above, the figure shows that $1+f\left(\alpha^{*}\right)=0$ is achieved for $\alpha^{*}$ extremely close to zero, the onset of synchronisation corresponds to degenerate $\alpha^{*}$, namely a saddle node bifurcation.

In our case with frustration parameters we have that $f(0)=f\left(0,\left\{\lambda_{i}\right\}\right)$ as well as $f^{\prime}(0)=f^{\prime}\left(0,\left\{\lambda_{i}\right\}\right)$. More precisely, one obtains

$$
f(0)=\frac{\sigma}{\sigma_{\omega}^{2}} \sum_{i} \omega_{i} k_{i} \sin \left(\lambda_{i}\right),
$$


and

$$
f^{\prime}(0)=\frac{\sigma}{\sigma_{\omega}^{2}}\left(\sum_{i} \omega_{i} \cos \lambda_{i} \sum_{j} A_{i j} \omega_{j}-\sum_{i} \omega_{i}^{2} k_{i} \cos \lambda_{i}\right) .
$$

We observe in this structure a weighted version of the Laplacian, with weights given by $\cos \lambda_{i}$, as already seen in Eq. (5).

High values of the order parameter $r$ may be obtained by minimising the intersection point of the non-linear function $1+f$ with the abscissa. Because of the $\lambda_{i}$ dependence we see an alternative to the scenario of [18], that this may be obtained by changing the frustrations to give values of $1+f(0)$ as close as possible to zero whilst also ensuring that $f^{\prime}(0)$ is as large as possible in magnitude and negative in sign. Simultaneously, an onset of synchronisation for low coupling is obtained if $1+f(0)$ is as small as possible for very small coupling strengths $\sigma$.

In Fig. 7 we illustrate results for $1+f(\alpha)$ for one realisation of a regular random small world network. Panel (a) of Fig. 7 shows that the tuning scheme can shift $1+f(0)$. In particular, $1+f(\alpha)$ is minimized if $\sin \lambda_{i}=-1$ for non-recruitable oscillators; however at the coupling of $\sigma=0.15$ no crossing of the abscissa is found yet. In panel (b) of Fig. 7 we see that increasing the value of coupling allows the lowest value of $1+f(\alpha)$ to drop below zero, indicating the onset of synchronisation via a saddle node bifurcation.

It also becomes apparent that the most important contributing factor towards finding solutions of $1+f(\alpha)=0$ for increasing coupling is a linear decrease in $1+f(0)$ corresponding to a linear increase in the number of recruitable oscillators with $\sigma$. Thus, in the frustrated model, the main contributing factor is reducing $1+f(0)$ rather than $f^{\prime}(0)$. We thus realise that, provided the coupling strength is larger than $\sigma_{r}$ given in Eq. (9), optimal synchronisation is obtained when the recruitment condition given in Eq. (7) is fulfilled, for which $r=1$. However, macroscopic synchronisation can already be achieved for $\sigma<\sigma_{r}$. In that case $1+f(0)$ can obviously be minimized by tuning recruitable oscillators according to Eq. (7) and setting frustration parameters of all non-recruited oscillators to $\lambda_{i}=-\operatorname{sgn} \omega_{i}$. For this particular realisation of the network and the three tuning schemes, onsets of synchronisation are predicted for $\sigma=0.306$ (ordinary Kuramoto), 0.228 (type I tuning), and 0.208 (type II tuning).

Having establish that tuning of the frustration parameters can significantly boost synchronisation, it is interesting to see how such tuning can be dynamically achieved. The next section presents a simple co-dynamics of frustration parameters and the ordinary Kuramoto dynamics which can allow for the emergence of tuned frustration parameters in certain parameter regions.

\section{CO-DYNAMICS OF FRUSTRATIONS AND ANGLES}

\section{A. Definition, equilibria and numerical solution}

Consider now the model

$$
\begin{aligned}
\dot{\theta}_{i}(t) & =\omega_{i}+\sigma \sum_{j} A_{i j} \sin \left(\theta_{j}(t)-\theta_{i}(t)+\mu_{i}(t)\right), \\
\dot{\mu}_{i}(t) & =\tau \sum_{j} A_{i j} \sin \left(\theta_{j}(t)-\theta_{i}(t)\right),
\end{aligned}
$$

where the parameter $\tau$ sets the time scale for the coevolution of the frustration parameters. A choice of $\tau=$ 0 reproduces the frustrated Kuramoto model, discussed above.

To study the equilibria of this system, consider the ansatz

$$
\begin{aligned}
\theta_{i}(t) & =\Omega t+\phi_{i}(t) \\
\mu_{i}(t) & =\lambda_{i}+\chi_{i}(t)
\end{aligned}
$$

where the fluctuations $\phi, \chi$ are regarded as small. Thus $\lambda_{i}$ represents the static limit of the $\mu_{i}(t)$ frustration codynamics.

Inserting the ansatz into Eqs. $(19,20)$ and expanding in fluctuations we again extract here the zeroeth order equation from the $\theta_{i}$ dynamics which turns out to be identical to Eq. (4) from the static system. We consider the fluctuations in detail shortly. For now, we see one key difference in the role of $\Omega$ between the static and coevolving systems, namely the role of a conservation law for the latter. Summing over $i$ in Eq. (20) gives

$$
\sum_{i} \dot{\mu}_{i}(t)=0 .
$$

(The $\theta_{i}$ enjoy no such conservation, as in the static frustrated system.) From this, the recruitment condition and assuming stability (the conditions for which are to be determined below), we obtain

$$
\sum_{i} \arcsin \left(\frac{\Omega-\omega_{i}}{\sigma k_{i}}\right)=\sum_{i} \mu_{i}(0)+2 n \pi
$$

with $n$ any integer due to the periodicity of the recruitment condition, Eq. (7). Thus $\Omega$ in the co-evolving system is effectively determined from the initial conditions for the $\mu_{i}$ (which were selected at random in simulations shown above) with the value of $n$ selected according to the number of jumps of oscillators around the circle by the time the equilibria are reached.

To give a first sense of the behaviour of the system of Eqs. $(19,20)$, we solve the equations numerically for a random regular graph of $N=50$ with degrees $k_{i}=4$ at coupling $\sigma=0.35$ and a specific realisation of frequencies drawn from $[-1,1]$, and initial conditions $\theta_{i}(0), \mu_{i}(0)$. In Fig. 8 we show plots for different $\tau$ of the individual oscillator phases $\theta_{i}(t)$ (grey curves), the dynamical frustration variables $\mu_{i}(t)$ (red curves), the equilibrium frustration 


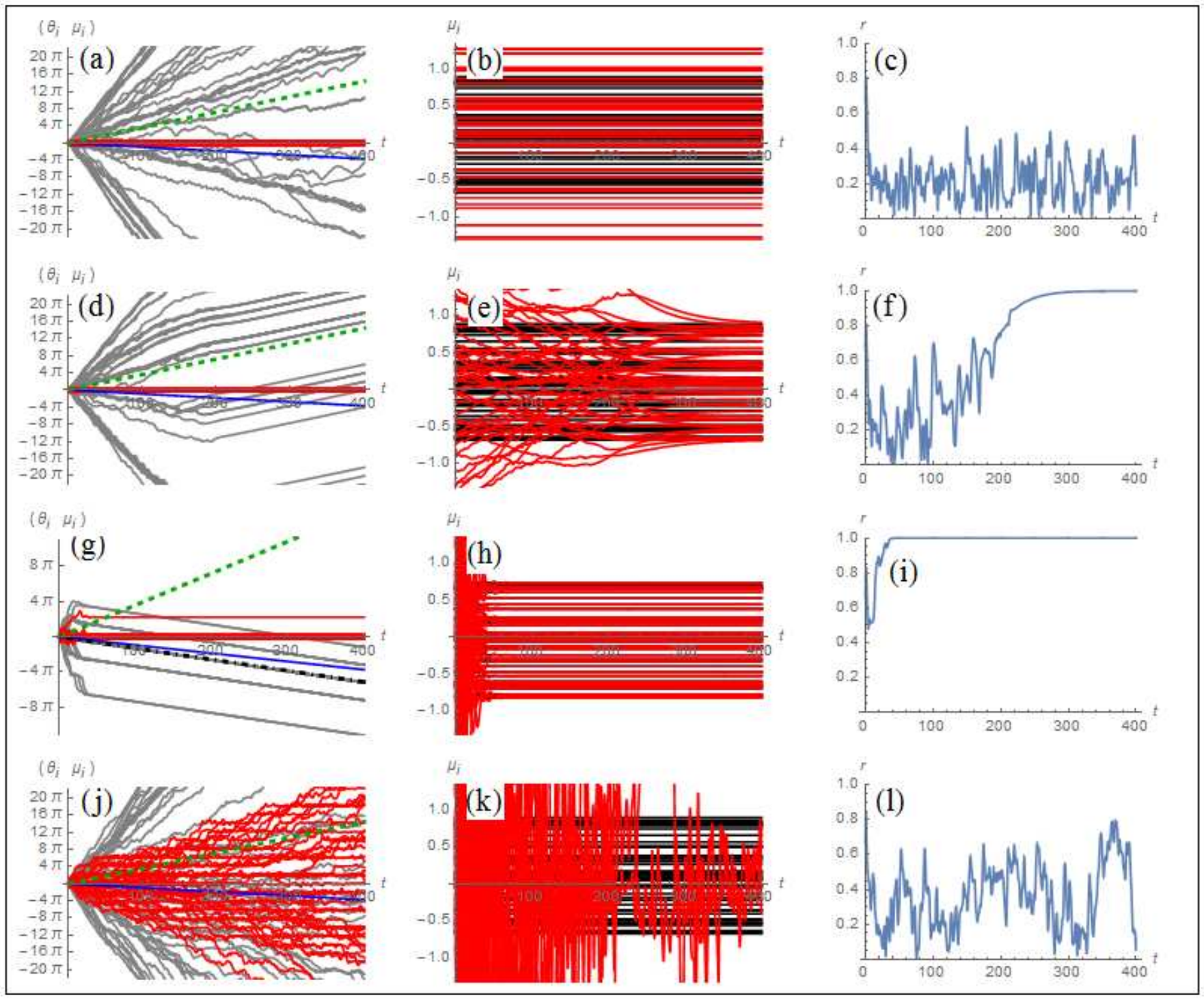

FIG. 8. Plots of $\theta_{i}$ (grey) and $\mu_{i}$ (red) on the left (a,d,g,j), the $\mu_{i}(t)$ (red) and fixed points $\lambda_{i}$ (black) in the middle (b,e,h,k), and the order parameter $r$ of Eq. (10) on the right (c,f,i,l) computed by numerically integrating the system Eqs. (19,20) for a Random Regular graph of $\langle k\rangle=4$ and $N=50$ and coupling $\sigma=0.35$. The same random seed has been applied to each case, with different $\tau$, namely $\tau=0$ (top), $\tau=0.005$ (second row), $\tau=0.2$ (third row) and $\tau=0.5$. On the left hand plots (a,d,g,j), we superimpose the trajectories $\bar{\omega} t$ (blue) and $\Omega t$ (dashed green and dot-dashed black), where $\Omega$ is solved from Eq. (23) with $n=0$ (dashed green) and $n=-1$ (dot-dashed black).

parameters $\lambda_{i}$ (black lines) from the recruitment condition for the $\Omega$ - itself solved from the initial conditions using Eq. (23) - and the order parameter $r(t)$. The same random seed is used to generate the particular graph, frequency and initial condition realisations. Superimposed on the $\theta_{i}, \mu_{i}$ trajectories in the left hand panels of Fig. 8 we show $\bar{\omega} t$ and $\Omega t$; for $\tau=0.2$ we show $\Omega t$ for choices of $n=0$ (dashed green line) and $n=-1$ (dot-dashed black line in the left hand third row plot labelled ' $\mathrm{g}$ ') in solving for $\Omega$.
We observe that for $\tau=0$ (top row, panels a,b and c) the $\mu_{i}$ assume constant values but poor synchronisation of the $\theta_{i}$, manifested in erratic behaviour of $r$. For $\tau=0.005$ (second row, panels $\mathrm{d}, \mathrm{e}$, and $\mathrm{f}$ ), the system synchronises - but not to $\bar{\omega} t$, rather to $\Omega t$ as seen in the match of the slope of the dashed green and grey trajectories in panel d. The tick marks of the vertical axis here emphasise that the parallel trajectories are $2 \pi$ copies of each other so that some trajectories have wrapped around the circle multiple times before synchronising into the 
pack. We observe in panel e at this value of $\tau$ that the $\mu_{i}(t)$ converge to the fixed point values $\lambda_{i}$, given by Eq. (7). Increasing to $\tau=0.2$ one first notices that synchronisation is maintained and initial transients become shorter (third row of panels in Fig. 8, panels $\mathrm{g}$, $\mathrm{h}$ and i). However, the $\theta_{i}$ trajectories converge to $\Omega t$, with $\Omega$ solved using $n=-1$ (black line in panel $\mathrm{g}$ ); computing the equilibria using this value of $\Omega$ shows convergence of the $\mu_{i}$ to these values in panel $\mathrm{h}$. Further increases in $\tau$ (bottom row, panels $\mathrm{j}, \mathrm{k}$ and $\mathrm{l}$ ) eventually lead to destruction of the synchronised state, in particular with erratic behaviour now evident in the $\mu_{i}$ (red trajectories in panels $\mathrm{j}$ and $\mathrm{k}$ ). With $\tau=0$, significantly higher $\sigma$ is required to enable the system to synchronise to the same degree as in the second and third rows of Fig. 8.

We see that the co-dynamical system allows for synchronisation at lower couplings, and at collective frequencies other than the mean, but also note that the timescale of the co-dynamics set by the parameter $\tau$ plays a crucial role in determining whether a fully synchronised state can be reached.

The observations just made for a particular network configuration are confirmed by examining the average order parameter against the coupling for larger systems using numerical simulation, displayed in Fig. 9, which also compares results to the ordinary (unfrustrated) $\mathrm{Ku}$ ramoto dynamics. The networks here are now random regular graphs of $N=500$ with degree $k_{i}=8$ and frequencies drawn from the uniform distribution between $[-1,1]$. The key feature in this plot is that the unfrustrated Kuramoto system lies in the middle range of the curves, with tuned and slow (small $\tau$ ) co-dynamics to the left, and fast (large $\tau$ ) co-dynamics to the right. These results confirm that the co-dynamics improves synchronisation compared to the ordinary Kuramoto dynamics. Moreover, increasing $\tau$ (namely, making the $\mu$-dynamics faster in comparison to the phase dynamics) strongly influences the synchronisation transition. By choosing small $\tau$ a substantial improvement in synchronisation is possible, while large $\tau$ impedes synchronisation such that it only occurs for larger coupling.

These results are reflected more specifically at the coupling value where $r$ deviates from zero: the critical couplings for small $\tau$ lie to the left in a pattern consistent with the position of the overall curves. In contrast, the coupling values where $r \approx 1$ nearly coincide for $0.001 \leq \tau \leq 0.1$, with then an appreciable jump in this value of $\sigma$ for $\tau=0.3$ and beyond. We shall return to this in our further examination of the linearisation next.

\section{B. Stability analysis}

Thus far we have derived the equilibria for the codynamical system, focusing on the conditions to zeroeth order in fluctuations around the static frustration condition and driving of oscillators to some frequency $\Omega \neq \bar{\omega}$. Now we test the stability of these configurations, using

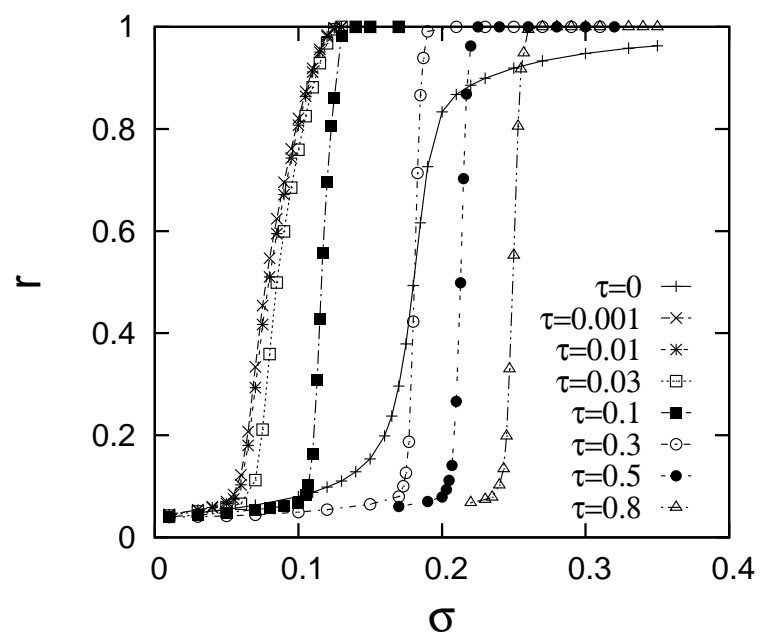

FIG. 9. Dependence of the order parameter $r$ on the coupling strength $\sigma$ for numerically integrated Kuramoto systems of 500 oscillators on a regular random graph with coordination number $k=8$. Compared are: (a) normal Kuramoto $(\lambda=0$, crosses), (b) $\lambda$-tuned Kuramoto (asterisks), and integration of the coupled system with dynamics for the phases and the lambda's for various choices of the timescale for the lambda dynamics $\tau=0.001-0.2$ (star, open and closed squares and circles). Native frequencies are drawn uniformly at random from $[-1,1]$.

ideas from [39], and explore the degree to which they explain some of the behaviours seen above.

The first order equations in fluctuations are:

$$
\begin{aligned}
& \dot{\phi}_{i}=-\sigma \sum_{j} \cos \lambda_{i} L_{i j} \phi_{j}+\sigma k_{i}\left(\cos \lambda_{i}\right) \chi_{i} \\
& \dot{\chi}_{i}=-\tau \sum_{j} L_{i j} \phi_{j}
\end{aligned}
$$

Here $L_{i j}$ is again the combinatorial Laplacian of the network. Note that the zeroeth order terms are not given here, with the recruitment condition Eq. (7) imposed strongly given that it defines the fixed point. Setting $\tau=0$ we recover in the first equation the weighted Laplacian with entries $\cos \lambda_{i}$, as mentioned in the first part of the paper. From this we confirm a sufficient condition for stability, that $\left|\lambda_{i}\right| \leq \pi / 2$. Forming a $2 N$ dimensional vector $v=(\vec{\phi}, \vec{\chi})$ enables Eqs. (24) to be written in terms of a 'super-Laplacian' $\mathcal{L}$. We denote the $2 N$ eigenvalues of this matrix by $\ell$ (since $\lambda$ is used for frustrations), but use $\ell^{(0)}$ to represent the $N$ eigenvalues of the standard Laplacian $L$.

To gain an initial crude analytic picture of the spectrum we assume - in contrast to our numerical computations thus far - that $\omega_{i}$ are matched to the degrees of the network, $k_{i}$, such that in the recruitment condition 
$\lambda_{i}=\lambda \forall i$. This leads to a form for the super-Laplacian

$$
\mathcal{L}_{i j}=\left(\begin{array}{cc}
\sigma(\cos \lambda) L_{i j} & -\sigma(\cos \lambda) \delta_{i j} k_{j} \\
\tau L_{i j} & 0
\end{array}\right) .
$$

We observe here that as $\lambda \rightarrow 0$ the ordinary combinatorial Laplacian emerges.

Expanding the $N$ variables $\phi_{j}$ in all $N$ eigenvectors of $L$, which, given knowledge of its spectrum, we label by $r=0, \ldots, N-1$ (since it has at least one zero mode).

$$
\mathcal{L}_{r j}=\left(\begin{array}{cc}
\sigma(\cos \lambda) \ell_{r}^{(0)} & -\sigma(\cos \lambda) k_{j} \\
\tau \ell_{r}^{(0)} & 0
\end{array}\right) .
$$

If we assume the network consists of one component there is exactly one zero eigenvalue of $L$, and so $\mathcal{L}$ has eigenvalues $\ell_{0}=0$ and

$$
\ell_{r j}=\frac{\sigma \ell_{r}^{(0)} \cos \lambda}{2}\left(1 \pm \sqrt{1-\frac{4 \tau k_{j}}{\sigma \ell_{r}^{(0)} \cos \lambda}}\right)
$$

for $r \neq 0$; note here we have used two indices for the spectrum as a consequence of this approximation scheme. Thus stability is guaranteed when $\cos \lambda>0$; for every $\lambda$ that is marginal, there will be an additional zero mode of $\mathcal{L}$. Secondly, for

$$
\tau>\sigma \ell_{r}^{(0)} \cos \lambda / 4 k_{j}
$$

the eigenvalues develop an imaginary part. Moreover, because $\ell_{r}^{(0)} \cos \lambda$ also appears in the real part, these modes that become complex will be associated with small real parts which are always negative. Thus here and in the following we are dealing with a system that is always stable but where, due either to negative real parts close to zero and/or imaginary parts, the equilibria may take a long time to reach with increasing oscillations.

To test this more realistically we numerically solve for the spectrum of the super-Laplacian for a situation closer to our numerical simulations: we choose an instance of the random regular graph of $N=500, k=8$ and an instance of frequencies drawn from $[-1,1]$. We set the coupling to $\sigma_{r}=0.125$ using Eq. (9), and the $\lambda_{i}$ using Eq. (7) (thus $\cos \lambda_{i} \geq 0 \forall i$ ), and set $\Omega=0$. In the top panel (a) of Fig. 10 we plot the imaginary part of each eigenvalue against its real part for various values of $\tau$. In the lower panel (b) we plot the eigenvalues in order of increasing absolute value but showing separately the real and imaginary parts. By inspection of the numerical values in the eigenvectors we have verified that the modes $i=1,500$ are dominated by $\theta$ excitations while for $i>500$ the eigenvectors are increasingly dominated by $\mu$ excitations.

We observe a number of features in the top plot (a). Firstly, the analytical prediction is verified: at every $\tau$ the spectrum involves negative real parts or zero eigenvalues (guaranteeing stability) but at very low $\tau$ eigenvalues are all negative real or zero (black points), while at $\tau=0.01$ the first eigenvalues with imaginary parts are associated with small negative real parts (orange points), with the rest lying along the negative real axis. Note that in this case there are two zero eigenvalues for every $\tau$ because we have chosen the coupling $\sigma$ exactly at the point, Eq. (9), where one $\lambda$ is marginally stable. Importantly, we observe that while eigenvalues are purely real there are gaps in the spectrum, especially observed for the black points in the inset of Fig. 10a, which 'fill in' once imaginary parts appear; we see that the real parts of the lowest values for $\tau=0.1$ (orange points) are smaller than a corresponding set of purely real negative eigenvalues. With larger $\tau$ all eigenvalues are all complex, with increasing value of their imaginary parts. The inset plot focuses on the lowest eigenvalues and emphasises that for the lowest lying non-zero mode the real parts do not change in magnitude but the imaginary parts increase as $\tau$ increases. Thus there is a threshold in $\tau$ below which all eigenvalues are purely negative real or zero. This is clearly seen in the lower plot (b) of the imaginary parts as functions of $\tau$ at small values, where in this instance at approximately $\tau=0.000035$ two oppositely signed imaginary parts appear; these modes are numbered 499 and 500 in the spectrum in terms of their order in absolute magnitude of the eigenvalue. Inspection of the eigenvector structure of these modes show these to be dominated by $\theta_{i}$ excitations. The main import of Fig. 10 is that as $\tau$ passes a threshold the number of modes with imaginary parts proliferates - starting with the low-lying modes.

The corresponding dynamical behaviour of fluctuations in this regime is thus that, though with $\cos \lambda \geq 0$ there is always stability, an increasing number of (predominantly) $\theta_{i}$ modes undergo long transients towards the equilibrium (due to the small negative real part). Associated with these same long transients are oscillations from the imaginary part, whose frequency increases with $\tau$, hindering (but never thwarting) the recovery of the synchronised state. That there is a threshold value of $\tau$ for which there is no imaginary part and a constant spectral gap (as seen in the inset of the top plot of Fig. 10) implies that there is no substantial change in the dynamics across these values. We therefore expect that for couplings allowing for near perfect synchronisation there should be no difference across a range of $\tau$ up to the threshold. This is consistent with the observed coincidence of the curves for the order parameter at $r \approx 1$ in Fig. 9 up to $\tau=0.1$.

\section{Basin of attraction}

Ultimately, the shift in the curves in Fig. 9 for different $\tau$ beyond $r \approx 1$ is a non-perturbative phenomenon, particularly the strong dependence of the critical coupling on $\tau$. We hypothesise that the cause of this change is a shrinking of the basin of attraction of the fixed point with increasing $\tau$. To test this we explore the influence of instantaneous perturbations on the synchronised state. Again for the $N=500$ random regular network of $k=8$ we simulate with very small $\tau=0.001$ and $\sigma=0.15$ 


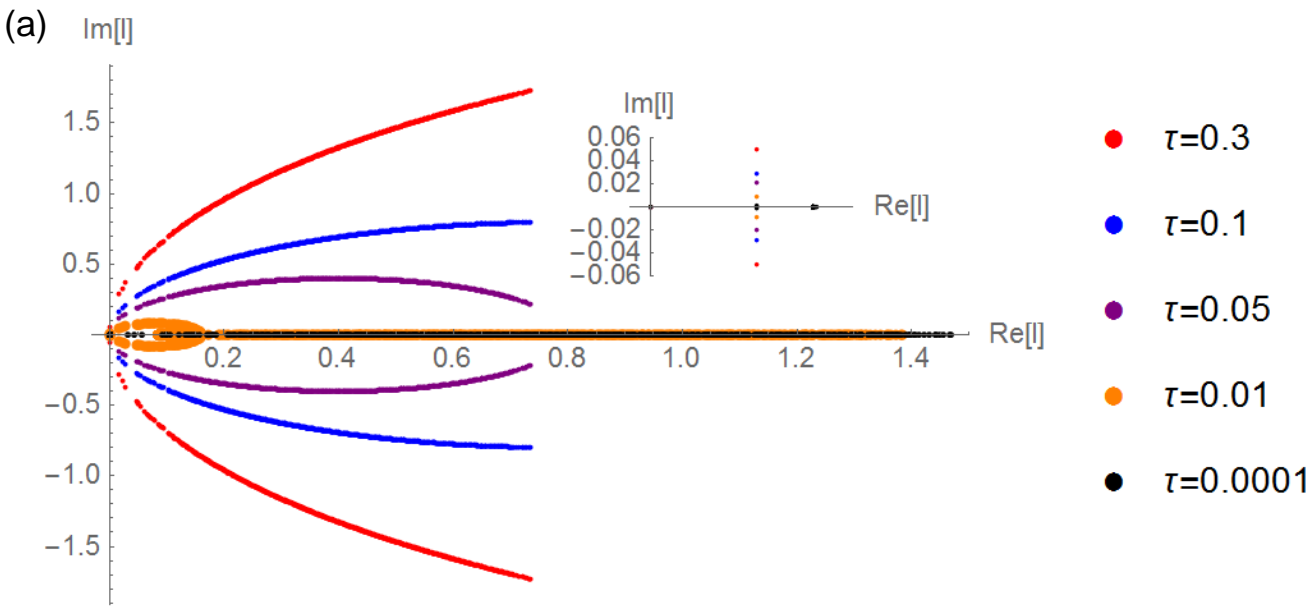

(b) $\operatorname{Im}\left(I_{i}\right)$

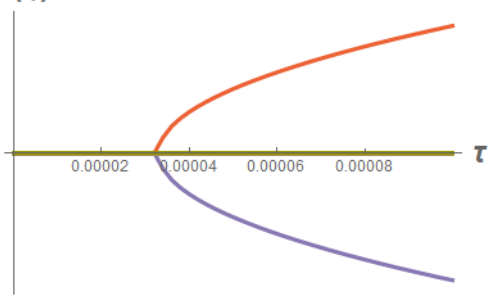

FIG. 10. (a) Eigenvalues of the super-Laplacian $\mathcal{L}$ for the Random Regular graph of $N=500,\langle k\rangle=8, \sigma=0.125$, plotting their imaginary part against the real part for different $\tau$, with inset zooming in to the origin, and (b) plotting the imaginary parts of all modes as a function of $\tau$ at small values where different colours represent different modes; only two have non-zero imaginary parts in this range seen in an upper and lower branch.

which guarantees that a perfectly synchronised state is attained. When this state is reached, at time $t=5000$, we perturb by adding to the state variables $\theta_{i}, \mu_{i}$ a random shift drawn uniformly at random from $[-\epsilon, \epsilon]$. We simultaneously change the value of $\tau$ and measure the recovery of the order parameter over time.

In Fig. 11 we plot time dependence of the order parameter, averaged over graphs, frequencies and perturbations for a given perturbation 'strength' $\epsilon$ and time scale $\tau$. In panel (a) we show the short time behaviour after the perturbation and in the lower the long term. We may make two observations from this result. For larger $\tau$ the system reverts more quickly back to the fully synchronised state or to partial synchronisation. If $\tau$ is too large, however, for an increasing number of configurations the system will no longer revert back to the fully synchronised state but move towards desynchronisation. The blue trajectory shown in panel (b) is an example of the latter. The inset plot in Fig. 11 shows that a histogram of final $r$ values across instances gives a bimodal distribution. The true average value of $r$ is then measured across these giving a result between 0 and 1 .

From such averaging we compute the average final order parameter $r$ for different values of the maximum perturbation strength, $\epsilon$, shown in Fig. 12. We see in panel (a) of Fig. 12 more clearly that for low $\tau$ as $\epsilon$ in- creases beyond some threshold there is a recovery from the perturbation only to a state of partial synchronisation, whereas for larger $\tau$ for arbitrary strength there is a complete loss of synchronisation. The sigmoidal nature of the curves indicates the presence of some critical perturbation strength beyond which that systems cannot recover from perturbations larger than that value. In panel (b) of Fig. 12 we show results where the perturbation is applied differently across the $\theta, \mu$ state variables: either identically for both ('combined') or for one or the other. The figure indicates that perturbations on the phases $\theta$ are the determining factor - the system is far more robust or insensitive to perturbations in the $\mu$ directions. This is not inconsistent with our observation close to synchronisation that the coincidence of imaginary parts with low-lying real parts in the spectrum of fluctuations from equilibrium occurs predominantly in the $\theta$ modes.

Motivated by the observation that values of $r \geq 0.9$ are typically associated with complete phase synchronisation, we finally obtain a proxy measure of the size of the basin of attraction by recording the intercept of the curves for $\epsilon$ in Fig. 12 with the line $r=0.9$ for different $\tau$ and perturbation regimes. This leads to the results shown in Fig. 13 which gives the dependence of this measure for the size of basins of attraction on the choice of the time scale parameter $\tau$. We note that choices of the 
(a)

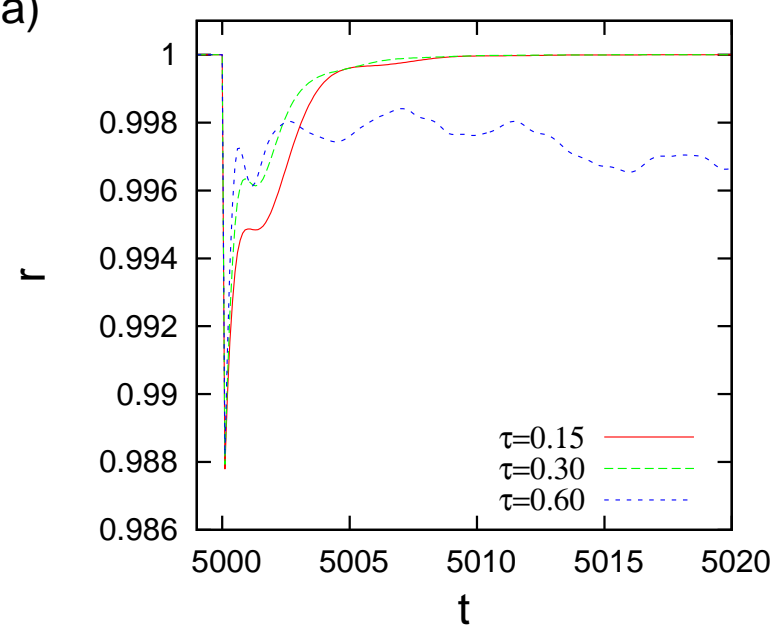

(b)

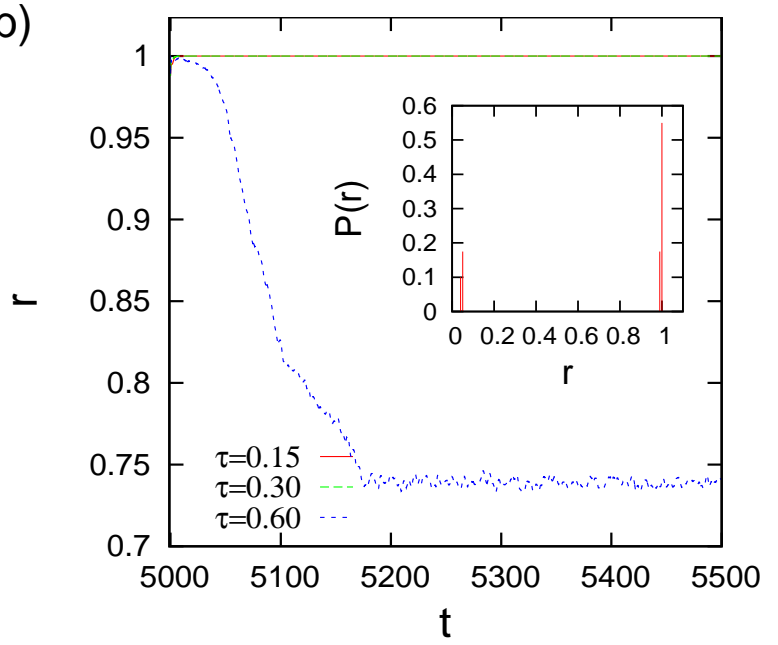

FIG. 11. Averaged order parameter behaviours (panel (a) - shortly after the perturbation, panel (b): longer term) for various $\tau$ after perturbing the system with noise of magnitude $\epsilon=0.3$ at time 5000. Inset in the lower panel is a histogram of final average order parameter values for $\tau=0.6$. Note that the curves for $\tau=0.15$ and $\tau=0.30$ overlap almost completely in the bottom panel.

intercept value of $r$ other than $r=0.9$ lead to qualitatively similar results. Regarding $\epsilon$ in Fig. 13 as the average size of the basin of attraction of the fully synchronised state we see that it shrinks with increasing $\tau$ moreso in the $\theta$ directions than the $\mu$.

This explains then the results in Fig. 9 that as $\tau$ increases the critical coupling increases: the basin of attraction of the synchronised state shrinks requiring larger coupling in order to compensate and allow the system to find the state starting from a random configuration. (a)

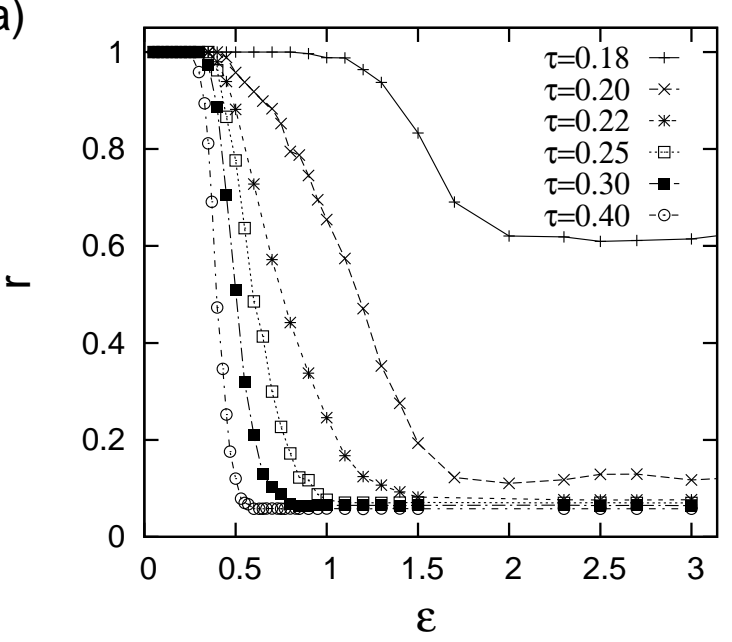

(b)

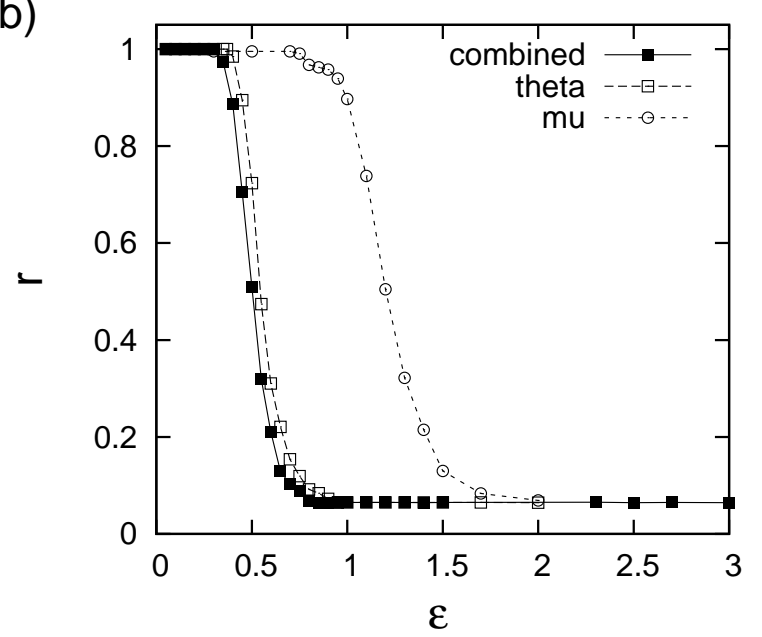

FIG. 12. Dependence of the final average $r$ on the maximum perturbation strength $\epsilon$ for various $\tau$ when perturbations are applied equally to all state variables (panel (a)), and applying in three different ways for $\tau=0.3$ (panel (b)), first equally to all state variables, second only to the phases $\theta$, and third only to the $\mu$.

\section{CONCLUSIONS}

Summarising our results, we have presented a model for local static and dynamic frustrations, or time delays, in the Kuramoto-Sakaguchi system on finite networks with random frequencies. Combining analytical and numerical studies we have shown that in the static model it is possible to optimise synchronisation - at lower couplings than for the ordinary Kuramoto model - by tuning frustrations using the local connectivity and frequency properties of a node, and setting non-recruitable nodes to a value consistent with marginal stability. In the time-dependent frustrated model, for small value of the time-constant for the frustrations the codynamics allow these to dynamically attain values consistent with 


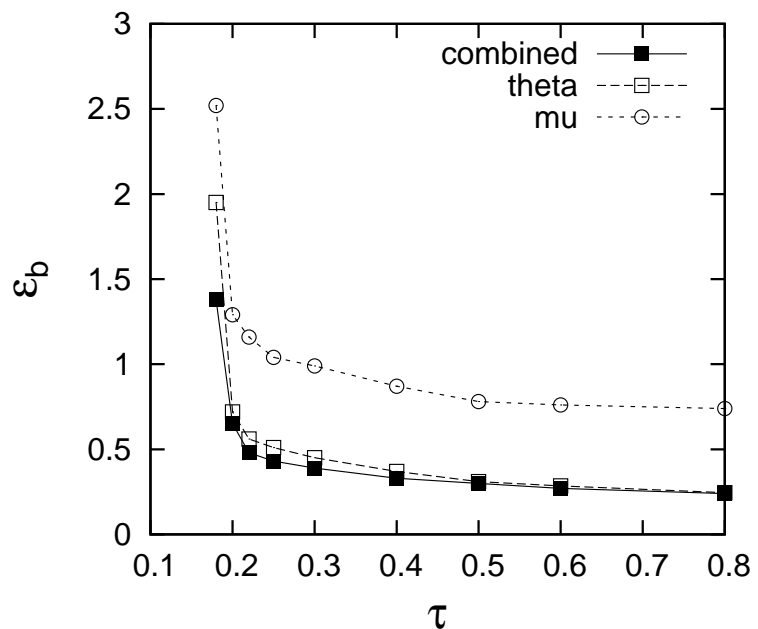

FIG. 13. Size of the basin of attraction as measured by the intercept in $\epsilon$ of the curves in Fig. 12 with the $r=0.9$ line plotted against $\tau$ for perturbations applied in both directions or in single directions as given in the legend.

recruitment. However, at larger time-constants the frustrations are hindered, at strong coupling, in finding the tuned state by rapid limit cycles which becomes, at values of coupling close to the critical point, a shrinking of the basin of attraction particularly for the phase modes. These behaviours are seen across a wide variety of networks and network sizes. We note a somewhat similar example of partially effective adaptivity in the case of coupled dynamical systems [40], where a constant $\gamma$ controls the speed of adaptation of link weights to the dynamics of the coupled oscillators; in some cases "too fast" an adaptation results in failure of synchronisation. Our basin of attraction approach may elucidate this behaviour there. Returning to the Kuramoto-Sakaguchi model, it is its extra complexity - the completely localised frustration parameters or variables - that, interestingly, has provided some scope for analytical approximation via linearisation to generate a threshold in coupling. Though it is not the critical coupling, it is a threshold that demarcates different dynamical regimes of behaviour visible in the numerical solutions. This is in contrast to the ordinary Kuramoto model where linearisation gives only a weak indication of the point for change in dynamical behaviour [33].

The beauty of this model lies in the novelty that by introducing additional disorder into the usual Kuramoto system, but allowing for a degree of adaptation through a codynamics, we may enhance order - manifested in synchronised behaviour. Moreover, the collective behaviour is no longer cursed by the mean of the limit cycle ensemble - but may be adjusted, up to a certain point, to operate faster or slower than the average. The application of these ideas lies in new mechanisms for 'controllability' or guidance of networked systems of heterogeneous agents. Certainly the Kuramoto model is quite a stylised representation of networked cycles. However, to the degree that it may be enfleshed in a real socio-technical system such tuned frustration or codynamics may also be adapted to a real world mechanism for enhanced cooperation in complex systems. The model presented in this paper also raises interesting questions for future research. For instance, it would appear of interest to study phase tuning in models of network control [41]: Does phase tuning oscillators influenced by external controllers ease control?

\section{ACKNOWLEDGEMENTS}

The authors are grateful for helpful discussions with Mathew Zuparic and constructive feedback from the referees. ACK is supported by a Chief Defence Scientist Fellowship and thanks the University of Southampton for its hospitality. No data sets are associated with this publication.
[1] Y. Kuramoto, Chemical Oscillations, Waves and Turbulence, Springer, Berlin, 1984

[2] J.A. Acebrón, L.L. Bonilla, C. Pérez-Vicente, F. Ritort and R. Spigler, The Kuramoto model: a simple paradigm for synchronization phenomena, Rev.Mod.Phys. 77, 137183, 2005

[3] S.N. Dorogovtsev, A.V. Goltsev and J.F.F. Mendes, Critical phenomena in complex networks, Rev.Mod.Phys. 80, 1276-1335, 2008

[4] A. Arenas, A. Díaz-Guilera, J. Kurths, Y. Moreno and C. Zhou, Synchronization in complex networks, Phys.Rep. 469 (3), 93-153, 2008

[5] F. Dörfler and F. Bullo, Synchronization in complex networks of phase oscillators: a survey, Automatica, 50 (6), 1539-1564, 2014
[6] S. Watanabe and S. H. Strogatz, Constants of motion for superconducting Josephson arrays, Physica D, 74, 197253,1994

[7] A. E. Motter, S. A. Myers, M. Anghel, and T. Nishikawa Spontaneous synchrony in power-grid networks, Nat. Phys., 9, 191-197, 2013

[8] S. De Monte, F. Ovidio, S. Dano, P. G. Sorensen, Dynamical quorum sensing: Population density encoded in cellular dynamics, Proc. Natl. Acad. Sci. U.S.A., 104, 18377, 2007

[9] L. Glass and M. C. Mackey From clocks to chaos: the rhythms of life, Princeton University Press, Princeton, 1988

[10] S. Yamaguchi, H. Isejima, T. Matsuo, R. Okura, K. Yagita, M. Kobayashi, and H. Okamura, Synchronization of cellular clocks in the suprachiasmatic nucleus, Science, 
302, 1408-1412, 2003

[11] H. Sakaguchi, Y. Kuramoto, A soluble active rotator model showing phase transitions via mutual entrainment, Prog.Theor.Phys.76, 576-581, 1986

[12] D. J. Watts and S. H. Strogatz, Collective dynamics of 'small-world' networks, Nature (London), 393, 440, 1999

[13] M. Brede, Phys. Lett. A, 372, 2618, 2008

[14] M. Brede, Eur. Phys. J. B, 62, 87, 2008

[15] L. Buzna, S. Lozano, and A. Díaz-Guilera, Phys. Rev. E, 80, 066120, 2009

[16] D. Kelly and G. A. Gottwald Chaos, 21, 025110, 2011

[17] P. S. Skardal, D. Taylor, and J. Sun, Phys. Rev. Lett., 113, 144101, 2014

[18] R. S. Pinto and A. Saa Phys. Rev. E., 92, 062801, 2015

[19] A.C.C. Coolen and C. Pérez-Vicente, Partially and frustrated coupled oscillators with random pinning fields, J.Phys.A: Math.Gen. 36, 4477-4508, 2003

[20] V. Nicosia, M. Valencia, M. Chavez, A. Díaz-Guilera and V. Latora, Remote synchronization reveals network symmetries and functional modules, Phys.Rev.Lett. 110, 174102, 2013

[21] C. Zhou and J. Kurths, Dynamical weights and enhanced synchronization in adaptive complex networks, Phys.Rev.Lett. 96, 164102, 2006

[22] S. Kirkland and S. Severini, alpha-Kuramoto partitions: graph partitions from the frustrated Kuramoto model generalise equitable partitions, Appl. Anal. Discr. Math. (9), 2015

[23] E. Barreto, B. Hunt, E. Ott, P. So, Synchronization in networks of networks: the onset of coherent collective behaviour of interacting populations of heterogeneous oscillators, Phys.Rev.E 77, 036107, 2008

[24] A.C. Kalloniatis, M.L. Zuparic, Fixed points and stability in the two-network frustrated Kuramoto model, Physica A 447, 21-25, 2016

[25] A. Pluchino, S. Boccaletti, V. Latora and A. Rapisarda, Andrea Rapisarda, Opinion dynamics and synchronization in a network of scientific collaborations, Phys.A: Stat.Mech. and its Appl. 372 (2), 316-325, 2006

[26] T. Mizumoto, T. Otsuka, K. Nakadai, T. Takahashi, K. Komatani, T. Ogata and H.G. Okuno, Human-robot ensemble between robot thereminist and human percussion- ist using coupled oscillator model, IEEE/RSJ Int. Conf. on Intelligent Robots and Systems, Taipei, Taiwan, 19571963, 2010

[27] B. Bollobás, Modern Graph Theory, Graduate Texts in Mathematics, Springer, New York, 1998

[28] P.S. Skardal, D. Taylor, J. Sun, A. Arenas, Erosion of synchronization in networks of coupled oscillators, Phys.Rev.E 91, 010802(R), 2015

[29] P.S. Skardal, D. Taylor, J. Sun, A. Arenas, Erosion of synchronization: coupling heterogeneity and network structure, Physica D 323-324, 40-48, 2016

[30] A.C. Kalloniatis, Ann.Phys. 348, 127-143, 2014

[31] Y. Maistrenko, B. Penkovsky, M. Rosenblum, Solitary state at the edge of synchrony in ensembles with attractive and repulsive interactions, Phys.Rev.E 89, 060901(R), 2014

[32] S. Gershgorin, Über die Abgrenzung der Eigenwerte einer Matrix, Izv.Akad.Nauk, USSR Otd. Fiz.-Mat. Nauk 6, 749-754, 1931

[33] A.C. Kalloniatis, From incoherence to synchronicity in the network Kuramoto model, Phys.Rev.E 82, 066202, 2010

[34] M.L. Zuparic and A.C. Kalloniatis, Stochastic (in)stability of synchronisation of oscillators on networks, Physica D 255, 35-51, 2013

[35] Y. Moreno and A. F. Pacheco, Eur. Phys. Lett. 68, 604, 2004

[36] H. Hong, M. Y. Choi, and B. J. Kim Phys. Rev. E. 65, 026139, 2002

[37] M. Brede Physica D 239, 1759, 2010

[38] G. A. Gottwald, Chaos, 25, 053111, 2015.

[39] L.M. Pecora and T.L. Carroll, Master stability functions for synchronized coupled systems, Phys.Rev.Lett. 80, 2109-2112, 1998

[40] C. Zhou, J. Kurths, Dynamical weights and enhanced synchronization in adaptive complex networks, Phys.Rev.Lett. 96, 164101, 2006

[41] Yang-Yu Liu, Jean-Jacques Slotine, and Albert-Lszl Barabsi, Controllability of complex networks, Nature 473, 167-173, 2011 